\title{
Electroacupuncture and Moxibustion Decrease Renal Sympathetic Nerve Activity and Retard Progression of Renal Disease in Rats
}

\author{
Josne C. Paterno ${ }^{a} \quad$ Cássia T. Bergamaschib $^{b}$ Ruy R. Campos ${ }^{b}$ Elisa M.S. Higa ${ }^{a}$ \\ Maria Fernanda Soares ${ }^{c}$ Nestor Schor ${ }^{a}$ Anaflávia O. Freire ${ }^{d}$ \\ Vicente Paulo Castro Teixeira ${ }^{a}$ \\ ${ }^{a}$ Nephrology Division, Department of Medicine, ${ }^{b}$ Cardiovascular Physiology Division, Department of Physiology, \\ ${ }^{c}$ Pathology Department, and ${ }^{\mathrm{d}}$ Chinese Medicine and Acupuncture Division, Department of Orthopedics and \\ Traumatology, Federal University of São Paulo (UNIFESP), São Paulo, Brazil
}

\section{Key Words}

Chronic kidney disease $\cdot$ Hypertension $\cdot$ Nitric oxide $•$

Renal sympathetic nerve activity Electroacupuncture $\cdot$

Moxibustion

\begin{abstract}
Background/Aim: Chronic kidney disease (CKD) is an increasing major public health problem worldwide. The sympathetic nervous system and nitric oxide play an important role in the pathogenesis of CKD. Traditional Chinese medicine has accumulated thousands of years of therapeutic experiences. Electroacupuncture (EA) and moxibustion (MO) are two such therapeutic strategies. The aim of this study was to investigate the renal and hemodynamic effects of EA-MO in an experimental model of a CKD. Methods: Male Wistar rats submitted to 5/6th nephrectomy (5/6 NX) were studied for 8 weeks. There were four groups: (1) control, normal rats; (2) NX, 5/6 NX only; (3) NX-AS, 5/6 NX and EA-MO session using sham points, and (4) NX-AM, 5/6 NX and EA-MO session using real acupoints. Biochemical and blood pressure studies, renal sympathetic nerve activity measurements, nitric oxide levels and the histopathological indices were assessed. Results: The EA- and MO-treated group pre-
\end{abstract}

\section{KARGER}

Fax +4161306 1234

E-Mail karger@karger.ch

www.karger.com
(C) 2012 S. Karger AG, Basel

1420-4096/12/0355-0355/\$38.00/0

Accessible online at:

www.karger.com/kbr sented significant improvement in all measured functional and histopathological parameters. Conclusion: These findings suggest that EA-MO had beneficial effects on CKD. This effect was probably achieved by the modulation of the renal sympathetic nerve activity and nitric oxide levels, leading to decreased blood pressure, which is associated with less proteinuria.

Copyright $\odot 2012$ S. Karger AG, Basel

\section{Introduction}

Although there have been many advances in the understanding of the pathophysiology of chronic kidney disease (CKD), the number of patients suffering from this disease is growing, and the demand for renal replacement therapy is increasing. Efforts employed against CKD cannot prevent progression of the disease, which culminates in end-stage disease despite application of the appropriate therapies [1]. Therefore, novel concepts and approaches are needed.

Over the last few decades, medical treatments based on traditional Chinese medicine (TCM) often rely on a combination of needle insertion and thermal stimula- 
tion to achieve results; the efficacy of such practices is increasingly recognized worldwide [2-4]. Electroacupuncture (EA) is the electrical stimulation of acupoints using acupuncture needles and a short current pulse at various frequencies [5]. Moxibustion (MO) is a technique that applies heat to acupoints by means of burning a compressed, powdered, combustible mass obtained from the young leaves of Eurasian artemisia (Artemisia vulgaris) $[6,7]$.

EA is increasingly accepted as a viable therapy for treating a variety of diseases and has been used to improve visceral autonomic malfunctions $[5,8]$. EA and MO stimulation have been used to modulate autonomic nervous activity, neurotransmitter levels, endogenous substance levels, levels of inflammatory factors and cardiovascular and renal function. It has also been used to treat mild to moderate hypertension [8-10].

CKD is becoming a worldwide public health problem [11]. The relevant pathogenetic factors contributing to the progression of CKD include high blood pressure, neurogenic factors and the decreased production of nitric oxide (NO) $[12,13]$. CKD may develop as a consequence of chronic hypertension and may also contribute to higher mortality in hypertensive patients $[13,14]$. In this respect, hypertension can be a cause of and a sustaining and worsening factor in the progression of renal disease. Evidence is accumulating that activation of the sympathetic nervous system (SNS) could play a major role in the progression of CKD $[15,16]$. Enhanced activity of the SNS has been demonstrated in patients with CKD $[16,17]$. This effect is, at least in part, a consequence of chronic activation of sympathoexcitatory inputs through renal afferent nerves within the failing kidneys because it is not seen in patients who have undergone bilateral nephrectomy [18].

In CKD patients, the overall production of $\mathrm{NO}$ is decreased, which contributes to cardiovascular events and further progression of CKD [19]. Although the mechanisms responsible for decreased NO production are unclear, potential explanations are reduced $\mathrm{NO}$ availability due to a state of increased oxidative stress or uncoupling of NO synthase due to cofactor deficiency [19-21].

In a previous study, we demonstrated that $\mathrm{EA}$ and $\mathrm{MO}$ attenuate progression of renal disease by decreasing mean arterial pressure (MAP) and proteinuria [22]. To further understand these effects, we evaluated the effects of $\mathrm{EA}$ and $\mathrm{MO}$ on the metabolism of $\mathrm{NO}$ and on renal sympathetic nerve activity (RSNA) in a rat model of CKD.

\section{Materials and Methods}

\section{Animals}

In this study, all experimental procedures were conducted according to the National Institutes of Health Guidelines for the Use and Care of Laboratory Animals, and the study protocol was approved by the Ethics in Research Committee of the Federal University of São Paulo (process No. 0720/09). Fifty-six male Wistar rats (250-300 g) were obtained from the animal care facility of our institution and were housed in a group cage, given access to rat chow and water ad libitum and maintained in a temperaturecontrolled environment $\left(23^{\circ} \mathrm{C}\right)$ on a 12 -hour light/dark cycle.

After a week adaptation period, the animals were weighed and transferred to metabolic cages kept in a humidity- and temperature-controlled room for $24 \mathrm{~h}$ to collect urine. The following day, 42 rats were randomly submitted to 5/6th nephrectomy (5/6 NX) under anesthesia with ketamine $(100 \mathrm{mg} / \mathrm{kg}$ i.p.) plus xylazine $(10$ $\mathrm{mg} / \mathrm{kg}$ i.p.). Briefly, after a ventral laparotomy, removal of the right kidney and ligation of two branches of the left renal artery were performed, resulting in the infarction of two-thirds of the left kidney.

\section{Experimental Protocols}

The experiments lasted 8 weeks. The study was carried out as two independent series of experiments, each composed of four groups: (1) control, normal rats; (2) NX, animals submitted to 5/6 NX only; (3) NX-AS, animals submitted to 5/6 NX and a 20-min EA-MO session using sham points twice weekly, and (4) NX-AM, animals submitted to 5/6 NX and a 20-min EA-MO session using real acupoints twice weekly. In the first series, MAP and RSNA measurements were assessed. In the second series, plasma and renal NO levels were assessed. Additionally, in both series, serum creatinine and urea, 24-hour proteinuria, tail-cuff blood pressure (TBP) and glomerulosclerosis and tubulointerstitial fibrosis were analyzed.

\section{Electroacupuncture and Moxibustion}

The localization of acupoints was based on rat anatomical references $[23,24]$. A number of papers studying different animal species have clearly shown that the location of acupuncture points follows a similar distribution in these mammals [25]. The entire treatment consisted of 16 sessions over the course of 8 weeks (20min sessions, twice weekly) $[26,27]$, under inhalatory anesthesia. The needle used for stimulation was $0.25 \mathrm{~mm}$ in diameter, $2 \mathrm{~cm}$ long and had a stainless steel stem and copper handle. For bilateral points, the positive output lead from the EA apparatus was connected to the left acupoint, and the negative terminal was connected to the right acupoint; the polarity between these points was continuously alternated.

The NX-AM group received acupuncture at points located on the hind limbs: ST-36 (Zusanli), KI-3 (Taixi). In the rat, ST-36 is located approximately $1 \mathrm{~mm}$ lateral to the tibial tuberosity, whereas KI-3 is located at the medial border of the tibia, $0.5 \mathrm{~mm}$ above the medial malleolus; both of these points are easily located by manual inspection. The needles were bilaterally inserted at a depth of approximately $0.5 \mathrm{~mm}$ at ST-36 and just into the skin at KI-3 (due to the lack of evident muscle mass under this area) and then subjected to EA $(20 \mathrm{~Hz} / 1 \mathrm{~V}$; Plexus AP585; VWV Biotherapy; Lautz, Brazil) for a period of $20 \mathrm{~min}$. In addition, MO stimulation was applied at the BL-23 (Shenshu) acupoint for a period of $2 \mathrm{~min}$, 
concomitant with the last 2 min of EA application. The stimulation time was the same for both groups. It is worthwhile mentioning that these points were mainly chosen because several studies reported their effects as anti-inflammatory and cardiovascular modulators [4, 7-9].

The NX-AS group received sham acupuncture at points located on neighboring skin areas ( $<1.5 \mathrm{~cm}$ from the real acupoints). The sham points were localized in regions with nerve distributions different from those present at ST-36, KI-3 and BL-23.

\section{Biochemical and Blood Pressure Studies}

Urinary protein excretion was measured using the Sensiprot Protein Assay Kit (Labtest Diagnostica, Brazil). Serum creatinine and urea were measured using the Labtest Creatinine and Urea Kits (Labtest Diagnostica). An initial baseline measurement of body weight and urine volume was made.

Indirect blood pressure was monitored in conscious rats with the tail-cuff method [28] (PE300; Narco Bio-Systems). Three to four measurements for each rat were obtained at each time once a week during the study. The week TBP was the median obtained from those measurements.

\section{Renal Sympathetic Nerve Activity}

For the MAP, the animals were anesthetized with urethane (1.2-1.4 g/kg i.p.), and the left femoral artery and vein were cannulated. The MAP was measured with a cannula inserted into the femoral artery that was connected to an on-line MacLab system (ADInstruments, Bella Vista, N.S.W., Australia) [29]. Supplemental doses of urethane $(10 \mathrm{mg} / \mathrm{kg}$ i.p.) were given as necessary to maintain an adequate depth of anesthesia, as assessed by the animals' lack of response to a noxious toe pinch, a respiratory pattern that followed the ventilator, and a stable blood pressure. Body temperature was monitored with a rectal probe (Letica Scientific Instruments) and was maintained between 36 and $37^{\circ} \mathrm{C}$ by a thermostatically controlled heating pad.

For the recording of RSNA, the left renal nerve was retroperitoneally exposed, placed on bipolar silver electrodes covered with mineral oil [30]. The signal from the renal nerve was displayed on an oscilloscope (TDS 220; Tektronix, Beaverton, Oreg., USA) and the nerve activity was amplified (gain 20,000, Neurolog; Digitimer, Welwyn Garden City, UK), filtered by a band-pass filter $(50-1,000 \mathrm{~Hz})$ and collected for display and later analysis using a PowerLab data acquisition system (ADInstruments). Intravenous phenylephrine bolus infusions $(10 \mu \mathrm{g} / \mathrm{kg})$ were administered to test the barosensitivity of the registered nerve. At the end of the experiments, the basal noise level of the renal sympathetic nerve was determined by the administration of the ganglion blocker hexamethonium (30 mg/kg, i.v.). RSNA was rectified online, integrated from the raw data obtained for each heart period and expressed as volts-seconds. Additionally, the neural activity was analyzed offline using the appropriate software (Spike Histogram; ADInstruments). The data are presented as the number of spikes per second (pps) [31].

\section{Nitric Oxide Levels}

At the end of the study period, animals were euthanized by decapitation for blood and renal tissue collection. Blood samples were collected in tubes containing EDTA (final concentration $2 \mathrm{mM}$ ) and $\mathrm{N}$-ethylmaleimide (final concentration $5 \mathrm{mM}$ ), immediately centrifuged at $+4^{\circ} \mathrm{C}(730 \mathrm{~g}, 5 \mathrm{~min})$ and stored at $-80^{\circ} \mathrm{C}$ for later analysis. The remnant kidney was rapidly excised, rinsed, sectioned and snap frozen in liquid nitrogen, and stored at $-80^{\circ} \mathrm{C}$ for later analysis.

Frozen renal tissue samples were cut in small pieces, placed in PBS buffer (1:5 w/v, pH 7.4) containing EDTA (2 mM) and Nethylmaleimide $(10 \mathrm{mM})$ and then immediately homogenized (BBX24 Bullet Blender, New York, USA). Supernatants were collected after centrifugation ( $15 \mathrm{~min}, 17,000 \mathrm{~g}, 4^{\circ} \mathrm{C}$ ).

Plasma and renal NO levels were determined by the chemiluminescence method using a Model 280 nitric oxide analyzer (Sievers Instruments Inc., Boulder, Colo., USA), which is a sensitive detector for measuring $\mathrm{NO}$ based on a gas phase chemiluminescent reaction between $\mathrm{NO}$ and ozone. The sensitivity for the measurement of $\mathrm{NO}$ and its reaction products is approximately 1 pmol [32].

\section{Histopathology}

Portions of the remnant kidneys were fixed in $10 \%$ neutral buffered formalin. Paraffin-embedded sections ( $3 \mu \mathrm{m}$ thick) were cut and stained with hematoxylin and eosin (HE), periodic acidSchiff's reaction and Masson's trichrome.

All morphologic evaluations were performed in a blinded manner by a single observer. Glomerular damage was evaluated on the basis of the percentage of glomeruli that were sclerotic or collapsed. Glomerulosclerosis was defined as segmental increases in the glomerular matrix, segmental collapse, obliteration of the capillary lumina, and accumulation of hyaline, often with a synechial attachment to Bowman's capsule [33, 34]. The extents of glomerulosclerosis and glomerular collapse were evaluated in each kidney by consecutive examination under light microscopy. Tubulointerstitial injury was defined as inflammatory cell infiltration, tubular dilation and/or atrophy, or interstitial fibrosis [21]. Injuries were examined in at least 20 areas using the following scoring system: $0=$ changes $<10 \%$ of the cortex sections; $1+=$ changes in up to $25 \%$ of the cortex sections; $2+=$ changes in up to $50 \%$ of the cortex sections; $3+=$ changes in $>50 \%$ of the cortex sections [11].

\section{Statistical Methods}

The results are presented as the mean \pm SD. Comparisons among different groups were evaluated using multiple analyses of variance (ANOVA) followed by a post hoc protected least-significant difference test. The level of statistical significance was defined as $\mathrm{p}<0.05$.

\section{Results}

\section{EA-MO Significantly Reduced Urine Volume, Serum Creatinine Levels and Urea Levels}

As shown in table 1, none of the experimental groups differed with respect to body weight at 8 weeks. The final urine volume was significantly higher compared to the starting volume in all of the nephrectomized groups. However, comparison of the final urine volume between the different groups showed that it was significantly reduced in the NX-AM group. 


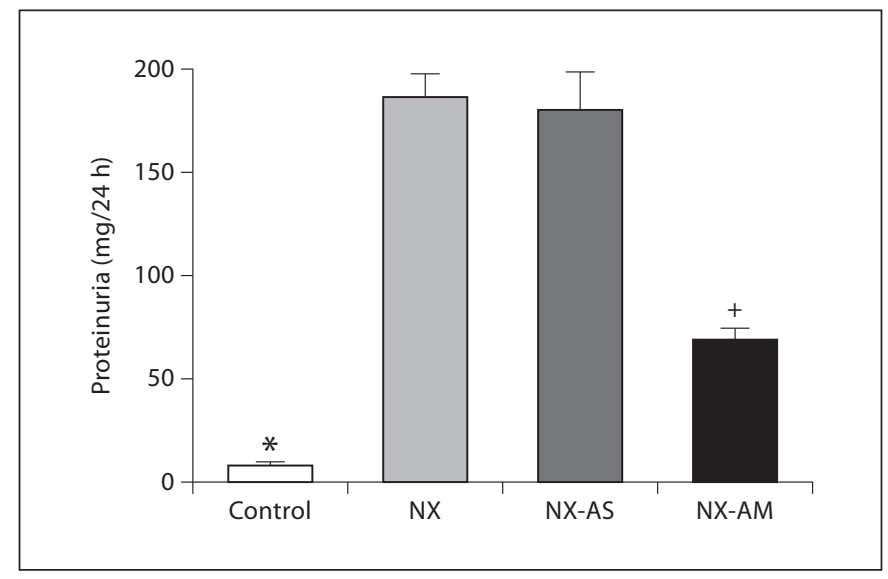

Fig. 1. Proteinuria $(\mathrm{mg} / 24 \mathrm{~h}):{ }^{*} \mathrm{p}<0.01$ vs. proteinuria $\mathrm{NX}, \mathrm{NX}-$ AS and NX-AM; ${ }^{+} \mathrm{p}<0.05$ vs. proteinuria NX and NX-AS.

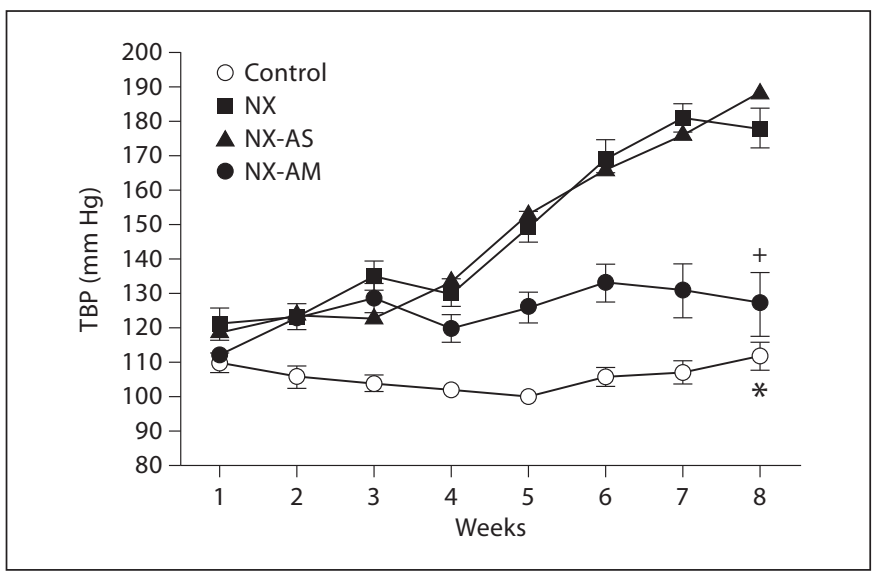

Fig. 2. TBP (mm Hg): * $\mathrm{p}<0.01$ vs. TBP NX and NX-AS; ${ }^{+} \mathrm{p}<0.05$ vs. TBP NX and NX-AS.

Table 1. Baseline and final functional and biochemical parameters

\begin{tabular}{|c|c|c|c|c|c|c|c|c|}
\hline \multirow[t]{2}{*}{ Group $(\mathrm{n}=7)$} & \multicolumn{2}{|c|}{ Body weight, $g$} & \multicolumn{2}{|c|}{ Diuresis, $\mathrm{ml} / 24 \mathrm{~h}$} & \multicolumn{2}{|c|}{ Serum urea, mg/dl } & \multicolumn{2}{|c|}{ Serum creatinine, $\mathrm{mg} / \mathrm{dl}$} \\
\hline & initial & end & initial & end & initial & end & initial & end \\
\hline NX & $275 \pm 9$ & $317 \pm 9$ & $11 \pm 1.5$ & $33.5 \pm 2.9^{*}$ & $40.5 \pm 1.3$ & $92.4 \pm 11.4^{*}$ & $0.4 \pm 0.2$ & $1.7 \pm 0.4^{*}$ \\
\hline NX-AS & $278 \pm 8$ & $322 \pm 10$ & $10 \pm 1.1$ & $34.6 \pm 2.7^{*}$ & $40.2 \pm 0.9$ & $95.1 \pm 19.4^{*}$ & $0.4 \pm 0.3$ & $1.5 \pm 0.2^{*}$ \\
\hline NX-AM & $281 \pm 5$ & $317 \pm 12$ & $12 \pm 0.5$ & $22.2 \pm 3.6^{*,+}$ & $40 \pm 1$ & $65.2 \pm 8.7^{*,+}$ & $0.4 \pm 0.3$ & $0.8 \pm 0.2^{*++}$ \\
\hline Control & $274 \pm 7$ & $338 \pm 10$ & $11 \pm 1.6$ & $13.5 \pm 1.2$ & $39.5 \pm 1.1$ & $42.3 \pm 1.9$ & $0.4 \pm 0.2$ & $0.4 \pm 0.8$ \\
\hline
\end{tabular}

Values are the means $\pm \mathrm{SD} .{ }^{*} \mathrm{p}<0.05$ vs. initial parameters; ${ }^{+} \mathrm{p}<0.05$ vs. final control, NX and NX-AS.

At the conclusion of the experiments, the serum creatinine and urea concentrations were significantly higher than the initial concentrations in all nephrectomized groups. However, the NX-AM group had significantly lower serum creatinine and urea concentrations than the other nephrectomized groups $(\mathrm{p}<0.05)$.

\section{EA-MO Significantly Reduced Proteinuria}

Proteinuria was significantly higher compared to control rats in all nephrectomized groups (fig. 1). However, rats that received EA-MO had significantly lower proteinuria compared to the rats in the other nephrectomized groups $(\mathrm{p}<0.05)$.

\section{Blood Pressure and RSNA Returned to Normal Values in NX-AM Rats}

At the end of study period, EA and MO treatment significantly attenuated the elevation of TBP and MAP ( $\mathrm{mm}$ $\mathrm{Hg}$ ) in the NX-AM relative to the NX or NX-AS groups $(\mathrm{p}<0.05)$ (fig. 2, 3). As shown in figures 4 and 5, EA and $\mathrm{MO}$ treatment significantly reduced RSNA in comparison to NX and NX-AS $(\mathrm{p}<0.05)$. The animals treated with EA and $\mathrm{MO}$ had a significant decrease in heart rate (HR) value compared to the other groups $(p<0.05)$ (fig. 5). Furthermore, there was no significant difference between the TBP, MAP and RSNA of the NX-AM and control group. Table 2 shows all the values for MAP, HR and RSNA.

\section{EA-MO Increased Serum and Renal NO Levels in Nephrectomized Animals}

Rats treated with EA and MO had a significant increase in serum and renal NO levels compared to the other nephrectomized groups ( $\mathrm{p}<0.01$ ) (fig. 6, 7).

\section{EA-MO Reduced Glomerulosclerosis and \\ Tubulointerstitial Fibrosis Indices}

As shown in figure 8, the glomerulosclerosis index (GSI) was significantly reduced in the EA-MO group 


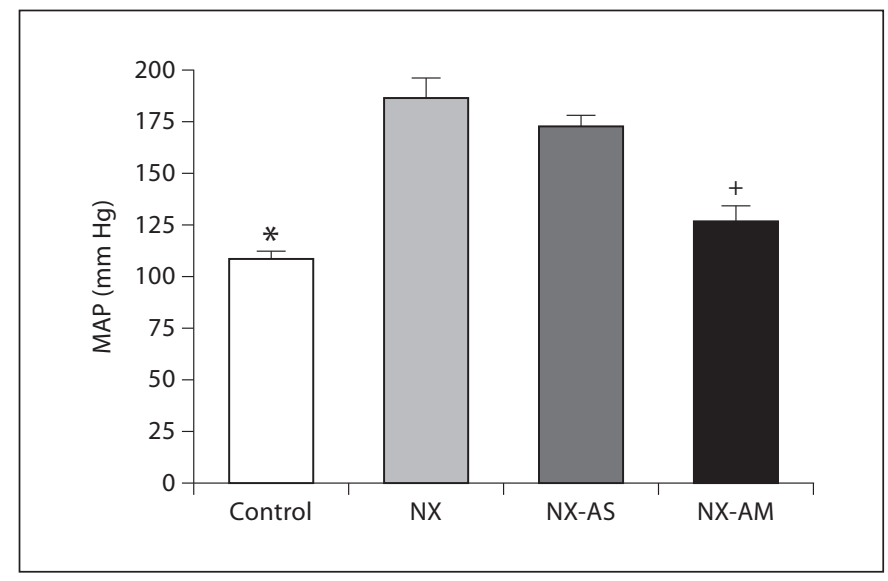

Fig. 3. MAP (mm Hg): * $\mathrm{p}<0.05$ vs. MAP NX and NX-AS; ${ }^{+} \mathrm{p}<$ 0.05 vs. MAP NX and NX-AS.

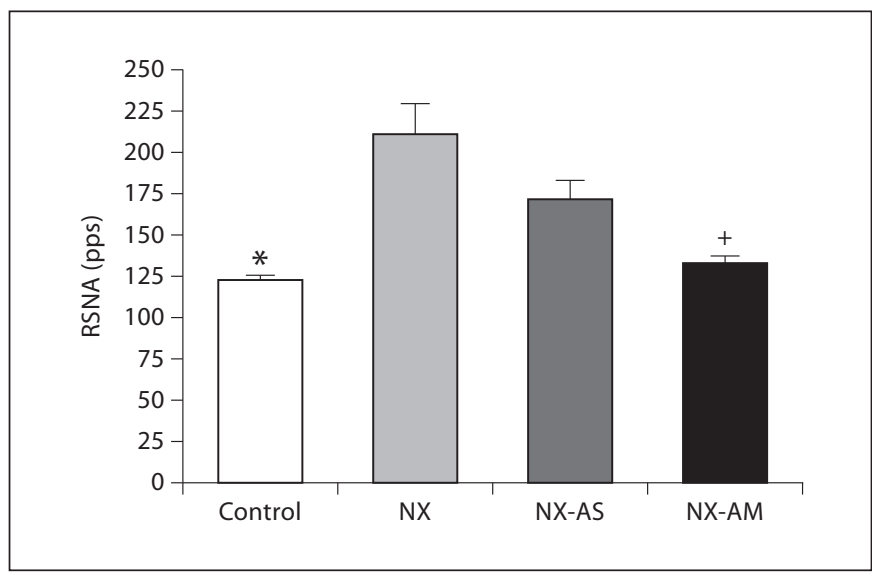

Fig. 4. RSNA (pps): ${ }^{*} \mathrm{p}<0.05$ vs. RSNA NX and NX-AS; ${ }^{+} \mathrm{p}<0.05$ vs. RSNA NX and NX-AS.

Table 2. MAP, HR, RSNA and NO levels

\begin{tabular}{|c|c|c|c|c|c|}
\hline Group & $\begin{array}{l}\text { MAP } \\
\mathrm{mm} \mathrm{Hg}\end{array}$ & $\begin{array}{l}\text { HR } \\
\text { bpm }\end{array}$ & $\begin{array}{l}\text { RSNA } \\
\text { pps }\end{array}$ & $\begin{array}{l}\text { Renal NO } \\
\mu \mathrm{M}\end{array}$ & $\begin{array}{l}\text { Serum NO } \\
\mu \mathrm{M}\end{array}$ \\
\hline NX & $187.5 \pm 22.2$ & $469 \pm 36$ & $211 \pm 46.5$ & $62.5 \pm 8.9$ & $66 \pm 13.7$ \\
\hline NX-AS & $172.3 \pm 15.2$ & $473 \pm 28$ & $170.1 \pm 28.2$ & $82.6 \pm 9.6$ & $60 \pm 14.7$ \\
\hline NX-AM & $127 \pm 17.7^{+}$ & $384 \pm 55^{+}$ & $131 \pm 12.2^{+}$ & $152 \pm 45.5^{+}$ & $163 \pm 42.2^{+}$ \\
\hline Control & $109.7 \pm 6.7^{*}$ & $481 \pm 52^{*}$ & $122 \pm 5.5^{*}$ & $104 \pm 18.6^{*}$ & $120 \pm 15.2^{*}$ \\
\hline
\end{tabular}

Values are the means $\pm \mathrm{SD} .{ }^{*} \mathrm{p}<0.05$ vs. NX and NX-AS; ${ }^{+} \mathrm{p}<0.05$ vs. NX and NX-AS.

compared to the other NX groups. In addition, the tubulointerstitial fibrosis index (TFI) grade of the NX-AM group was much lower than that of the other NX groups (fig. 9, 10).

\section{Discussion}

TCM has been increasingly recognized as an effective therapeutic approach in several fields of medicine. MO and EA have been used in TCM for centuries in the treatment of various diseases, including cardiovascular diseases and diseases involving hypertensive syndromes [7, 35, 36]. Recently, we demonstrated that EA-MO hindered the progression of CKD with reduced SBP/MAP and proteinuria [22].

In the present study, we confirmed our previous results that demonstrated the beneficial effects of EA-MO on the progression of renal diseases, whereby treated an- imals preserved concentration capacity and had normal SBP/MAP values, reduced proteinuria, normal serum creatinine and urea levels and better histopathological parameters. The current study also made significant progress by further elucidating the mechanisms of the specific effect on SBP/MAP.

It is already well established that hypertension is very common in patients with CKD and plays a key role in the progressive deterioration of renal function. Traditionally, hypertension in CKD was viewed as largely volume-dependent, but now there is increasing evidence that the activation of the SNS often accompanies CKD and, therefore, could contribute to elevation of arterial pressure, including its influence on fluid retention. The beneficial effect of EA-MO on blood pressure has been confirmed by several clinical and experimental studies described in the literature [7, 35-41].

Experimental evidence suggests that different pathophysiological mechanisms contribute to the progression 
Fig. 5. Representative traces of blood pressure (PAP), HR, MAP and RSNA. RSNA was amplified 20,000 times.

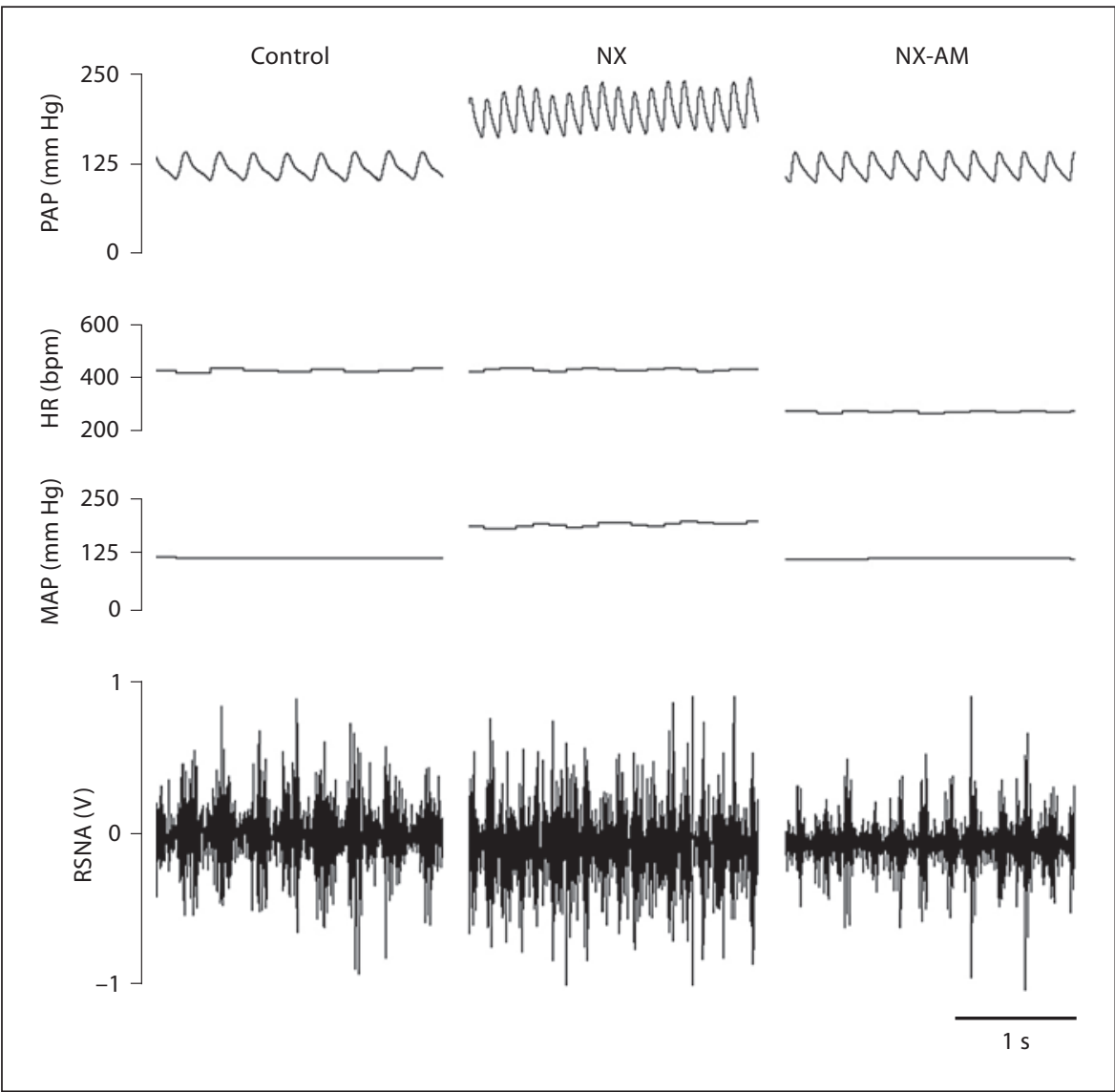

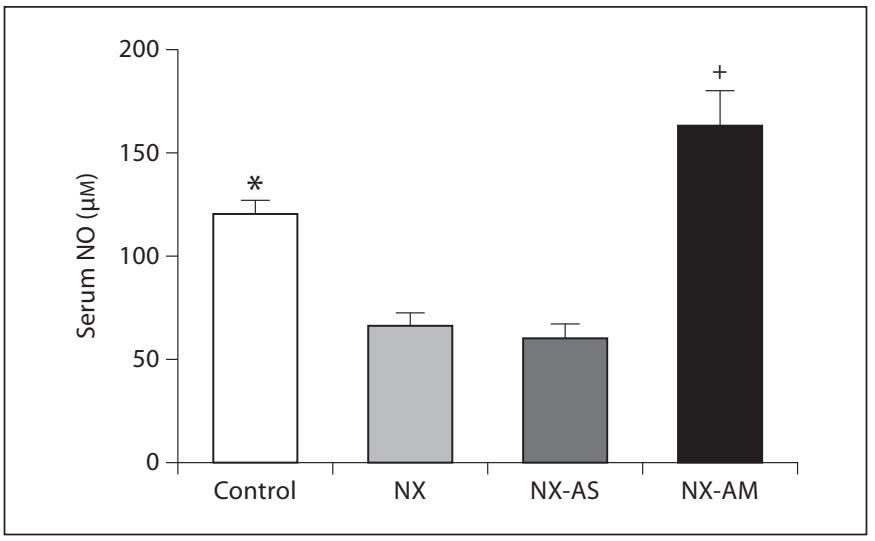

Fig. 6. Serum NO $(\mu \mathrm{M}):{ }^{*} \mathrm{p}<0.05$ vs. serum NO NX, NX-AS and NX-AM; ${ }^{+} \mathrm{p}<0.01$ vs. serum NO NX and NX-AS.

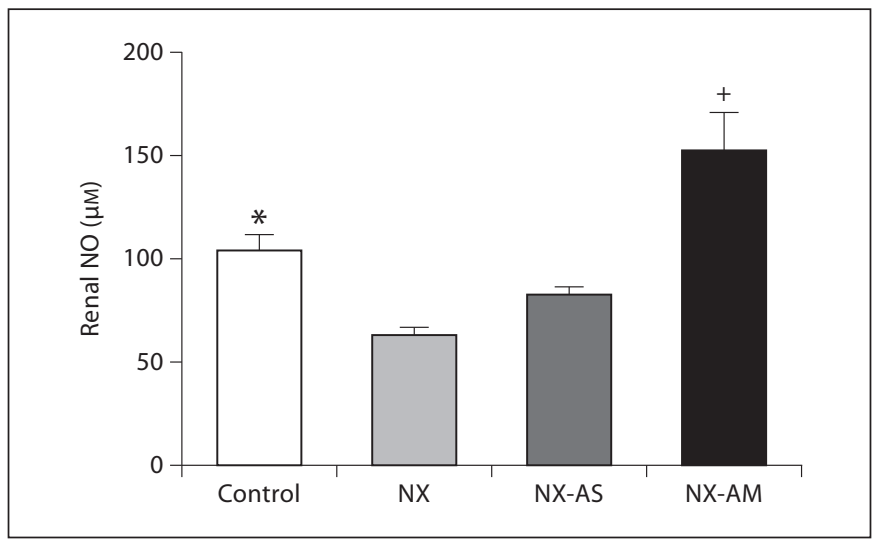

Fig. 7. Renal NO $(\mu \mathrm{M}):{ }^{*} \mathrm{p}<0.05$ vs. renal NO NX, NX-AS and $\mathrm{NX}-\mathrm{AM} ;{ }^{+} \mathrm{p}<0.01$ vs. renal NO NX and NX-AS. 


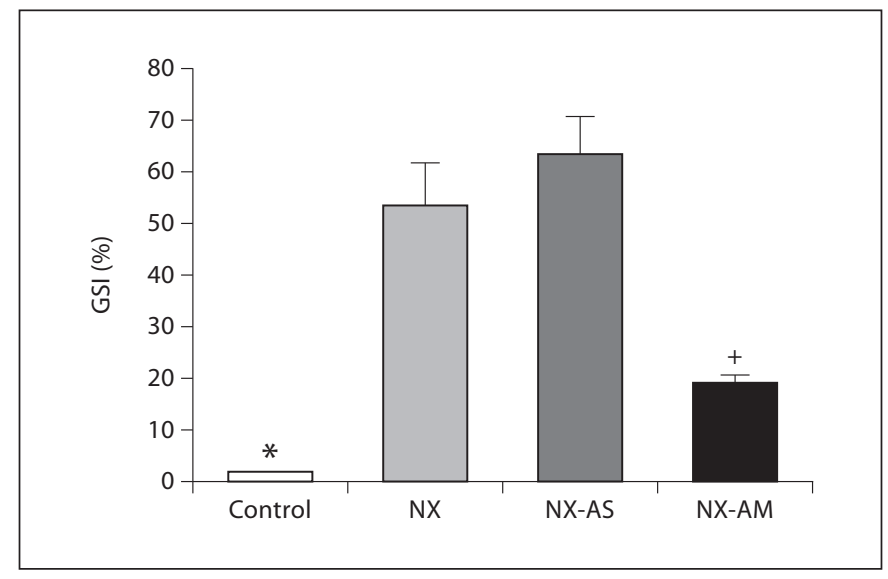

Fig. 8. GSI (\%): ${ }^{*} \mathrm{p}<0.01$ vs. GSI NX and NX-AS; ${ }^{+} \mathrm{p}<0.05$ vs. GSI NX and NX-AS.

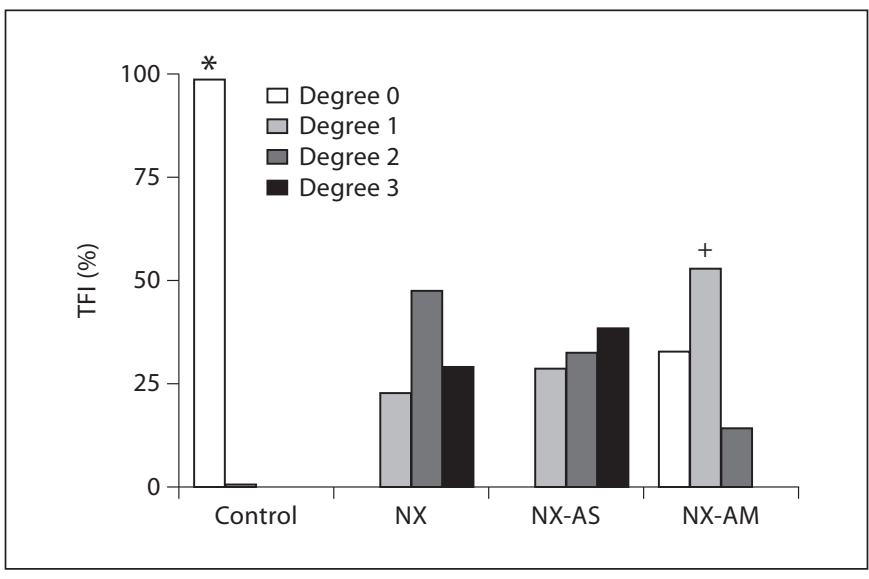

Fig. 9. TFI (\%): ${ }^{*} \mathrm{p}<0.01$ vs. TFI NX and NX-AS; ${ }^{+} \mathrm{p}<0.05$ vs. TFI NX and NX-AS.

Fig. 10. Histological analysis of the kidney from experimental group rats by Masson's trichrome staining: NX (a), NX-AS (b), NX-AM (c) and control (d). Treatment with EA-MO (c) had significantly less renal injury and widespread glomerulosclerosis and tubulointerstitial injury can be seen in both NX and NX-AS rats $(\mathbf{a}, \mathbf{b})$. Masson's trichrome. $\times 40$.

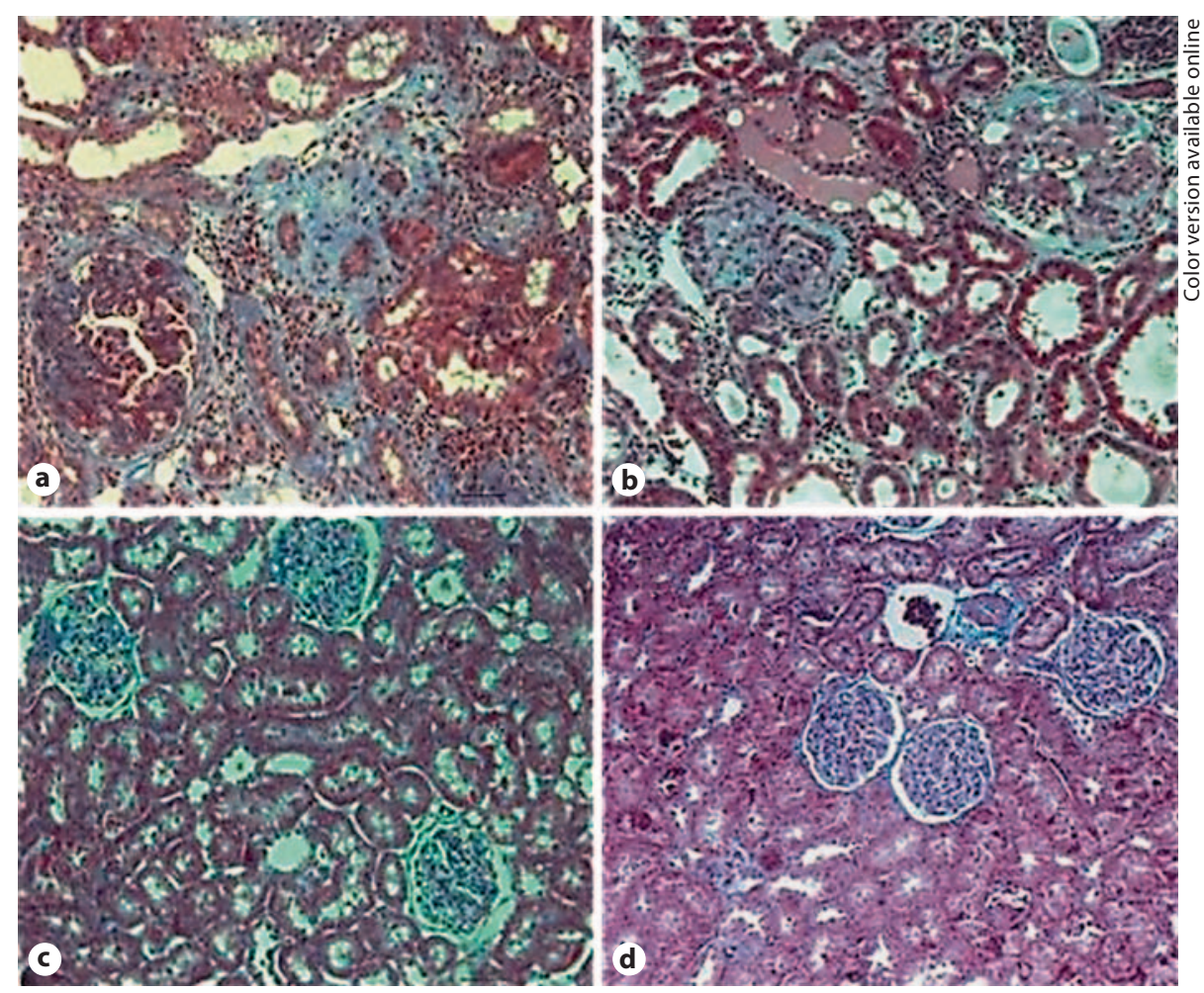

of renal disease, including hypertension [42], sympathetic activation, renin-angiotensin-aldosterone system activation, proteinuria, oxidative stress, inflammatory cell infiltration and action of various mediators and growth factors and cytokines, including NO and TGF- $\beta_{1}[1,43,44]$.

Sympathetic activation plays an important and distinctive role in hypertension and target organ damage associated with CKD. Experimental studies with 5/6 NX rats showed an increase in renal sympathetic activity by increasing the production of plasma norepinephrine, local release of noradrenaline and AII [13, 45]. Consistent with these studies, our nephrectomized animals without treatment had a significant increase in RSNA when compared with control animals. On the other hand, animals 
treated with EA-MO presented RSNA values in the normal range. Accordingly, Campese et al. [45] showed that remnant kidney rhizotomy promoted retardation of CKD with decreased MAP and histological improvement. Furthermore, patients with hypertension and non-dialysis CKD have been shown to exhibit hyperactivity of the SNS by using direct detection of peripheral sympathetic activity with microneurography and by high plasma levels of norepinephrine [16]. Thus, reduction in sympathetic activity may assist in antihypertensive treatment [46] and in CKD. Recently, Schlaich et al. [47] suggested that renal sympathetic nerve ablation appeared to be a logical therapeutic approach for treatment of hypertension.

The available data suggest that afferent signals from diseased kidneys to integrative structures in the brain result in activation of sympathetic outflow, stimulation of the renin-angiotensin-aldosterone system and alterations in the L-arginine/NO pathway and aggravation of hypertension [48]. In the central nervous system, the rostral ventrolateral medulla (RVLM) is an important part of the sympathetic efferent limb of cardiovascular reflex activity and, as such, it is important in maintenance of arterial blood pressure [31]. Interestingly, in many of these studies, EA appears to inhibit sympathetic effects by inhibition of sympathoexcitatory RVLM neurons [4951]. In a series of very important experimental studies, Longhurst and colleagues $[35,52,53]$ showed that EA inhibition of cardiovascular sympathetic neurons is mediated by a complex central nervous system response which, in turn, inhibits the activity of pre-motor sympathetic neurons in the RVLM.

Another possible explanation for the SNS modulation by EA-MO is an activation of opioid receptors in the central nervous system and increased production and secretion of endogenous opioids, serotonin, catecholamines, angiotensin II, and NO pathway [5, 8, 54]. Additionally, glutamate, acetylcholine, opioids, GABA, nociceptin, serotonin and endocannabinoids all appear to participate in the EA hypotensive response $[35,55,56]$.

The hypertension pathogenesis has also been associated with decreased NO production, which mediates arteriolar vasodilatation, reduces peripheral resistance, and lowers blood pressure. In the present study, we found that nephrectomized rats presented lower serum and renal NO levels in comparison to control rats. In contrast, EAMO treatment increased serum and renal NO levels above the normal levels. Consistent with our findings, it has been shown that total $\mathrm{NO}$ production is decreased due to impaired endothelial and renal $\mathrm{NO}$ production in CKD; thus, $\mathrm{NO}$ deficiency could play a role in CKD progression [57-59]. In addition, several studies have demonstrated that EA can modulate the production of $\mathrm{NO}$ synthases in hypertensive animals [60-62].

Taken together, we postulate that the reduced RSNA and the increased serum and renal NO levels caused by EA-MO treatment exerted an evident beneficial influence on SBP/MAP. This effect associated with reduced proteinuria improved renal function of EA-MO-treated animals, as demonstrated by the reduction of glomerulosclerosis serum creatinine and urea, preserved urinary concentration capacity and lower degrees of glomerular and tubular injury.

The value of EA and MO as effective therapeutic tools has been confirmed by numerous studies in recent decades. Scientific advances have clarified the neurobiological basis of both EA and MO, mainly through experimental models using EA at the acupoint ST-36 [27, 63]. To our knowledge, our work was the first to use EA at both ST-36 and KI-3 combined with MO in BL-23. However, further studies are needed in order to consolidate TCM concepts in nephrology as a valid adjunct treatment for CKD.

\section{Acknowledgments}

The excellent technical assistance of Margaret Gori, Clara Versolato Rasviskas, and Luciana Cristina Teixeira is gratefully acknowledged. This study was funded by the CNPq.

\section{Disclosure Statement}

The authors have no conflicts of interest to disclose.

\footnotetext{
References

1 Fogo AB: Mechanisms of progression of chronic kidney disease. Pediatr Nephrol 2007;22:2011-2022.

-2 Tabosa A, Yamamura Y, Forno ER, Mello LE: A comparative study of the effects of electroacupuncture and moxibustion in the gastrointestinal motility of the rat. Dig Dis Sci 2004; 49:602-610.

-3 Ahn AC, Kaptchuk TJ: Advancing acupuncture research. Altern Ther Health Med 2005; 11:40-45.

$\checkmark 4$ Lee S, Lee MS, Choi JY, Lee SW, Jeong SY, Ernst E: Acupuncture and heart rate variability: a systematic review. Auton Neurosci 2010;155:5-13.
} 
-5 Sugai GC, Freire AO, Tabosa A, Yamamura Y, Tufik S, Mello LE: Serotonin involvement in the electroacupuncture- and moxibustion-induced gastric emptying in rats. Physiol Behav 2004;82:855-861.

6 Kawakita K, Shinbara H, Imai K, Fukuda F, Yano T, Kuriyama K: How do acupuncture and moxibustion act? Focusing on the progress in Japanese acupuncture research. J Pharmacol Sci 2006; 100:443-459.

7 Kim JI, Choi JY, Lee H, Lee MS, Ernst E: Moxibustion for hypertension: a systematic review. BMC Cardiovasc Disord 2010;10:16.

8 Ma SX: Neurobiology of acupuncture: toward CAM. Evid Based Complement Alternat Med 2004;1:41-47.

-9 Cabýoglu MT, Ergene N, Tan U: The mechanism of acupuncture and clinical applications. Int J Neurosci 2006;116:115-125.

10 Garcia GE, Ma SX, Feng L: Acupuncture and kidney disease. Adv Chronic Kidney Dis 2005;12:282-291.

-11 Remuzzi A, Gagliardini E, Sangalli F, Bonomelli M, Piccinelli M, Benigni A, Remuzzi G: ACE inhibition reduces glomerulosclerosis and regenerates glomerular tissue in a model of progressive renal disease. Kidney Int 2006;69:1124-1130.

12 Wardle N: Glomerulosclerosis: the final pathway is clarified, but can we deal with triggers? Nephron 1996;73:1-7.

13 Campese VM: Neurogenic factors and hypertension in chronic renal failure. J Nephrol 1997; 10:184-187.

14 Khosla N, Bakris G: Lessons learned from recent hypertension trials about kidney disease. Clin J Am Soc Nephrol 2006; 1:229-235.

-15 Rump LC, Amann K, Orth S, Ritz E: Sympathetic overactivity in renal disease; a window to understand progression and cardiovascular complications of uremia? Nephrol Dial Transplant 2000;1735-1738.

-16 Tinucci T, Abrahão SB, Santello JL, Mion D Jr: Mild chronic renal insufficiency induces sympathetic overactivity. J Hum Hypertens 2001:15:401-406

17 Neumann J, Ligtenberg G, Klein II, Koomans HA, Blankestijn PJ: Sympathetic hyperactivity in chronic kidney disease: pathogenesis, clinical relevance, and treatment. Kidney Int 2004;65:1568-1576.

18 DiBona G: Dynamic analysis of patterns of renal sympathetic nerve activity: implications for renal function. Exp Physiol 2005; 159-161.

19 Passauer J, Pistrosch F, Bussemaker E: Nitric oxide in chronic renal failure. Kidney Int 2005;67:1665-1667.

20 Passauer J, Pistrosch F, Bussemaker E, Lässig G, Herbrig K, Gross P: Reduced agonist-induced endothelium-dependent vasodilation in uremia is attributable to an impairment of vascular nitric oxide. J Am Soc Nephrol 2005; 16:959-965.
21 Duffy SJ, Biegelsen ES, Eberhardt RT, Kahn DF, Kingwell BA, Vita JA: Low-renin hypertension with relative aldosterone excess is associated with impaired NO-mediated vasodilation. Hypertension 2005;46:707-713.

22 Paterno JC, Freire AO, Soares MF, Franco MF, Schor N, Teixeira VP: Electroacupuncture and moxibustion attenuate the progression of renal disease in 5/6 nephrectomized rats. Kidney Blood Press Res 2008;31:367373.

23 Murase K, Kawakita K: Diffuse noxious inhibitory controls in anti-nociception produced by acupuncture and moxibustion on trigeminal caudalis neurons in rats. Jpn J Physiol 2000;50:133-140.

24 Yin CS, Jeong HS, Park HJ, Baik Y, Yoon MH, Choi CB, Koh HG: A proposed transpositional acupoint in a mouse and rat model. Res Vet Sci 2008;84:159-165.

25 Dos Santos JG Jr, Tabosa A, do Monte FH, Blanco MM, de Oliveira Freire A, Mello LE: Electroacupuncture prevents cognitive deficits in pilocarpine-epileptic rats. Neurosci Lett 2005;384:234-238.

26 Vanderploeg K, Yi X: Acupuncture in modern society. J Acupunct Meridian Stud 2009; 2:26-33.

27 Freire AO, Sugai GC, Blanco MM, Tabosa A, Yamamura Y, Mello LE: Effect of moxibustion at acupoints Ren-12 (Zhongwan), ST-25 (Tianshu), and ST-36 (Zuzanli) in the prevention of gastric lesions induced by indomethacin in Wistar rats. Dig Dis Sci 2005;50: 366-374.

28 Ikeda K, Nara Y, Yamori Y: Indirect systolic and mean blood pressure determination by a new tail cuff method in spontaneously hypertensive rats. Lab Anim 1991;25:26-29.

29 Hirotsu C, Andersen ML, Bergamaschi CT, Tenorio NM, Araujo P, Tufik S: Sleep pattern in an experimental model of chronic kidney disease. Am J Physiol Renal Physiol 2010; 299:1379-1388

30 De Oliveira-Sales EB, Nishi EE, Boim MA, Dolnikoff MS, Bergamaschi CT, Campos RR: Chronic antioxidant treatment improves arterial renovascular hypertension and oxidative stress markers in the kidney in Wistar rats. Am J Hypertens 2010;23:473480.

-31 De Oliveira-Sales EB, Nishi EE, Boim MA, Dolnikoff MS, Bergamaschi CT, Campos RR: Upregulation of AT1R and iNOS in the rostral ventrolateral medulla is essential for the sympathetic hyperactivity and hypertension in the 2K-1C Wistar rat model. Am J Hypertens 2010;23:708-715.

32 Hamplh, V, Waters CL, Archer, SL: Determination of nitric oxide by the chemiluminescence reaction with ozone; in Feelisch M, Stamler JS (eds): Methods in Nitric Oxide Research. Chichester, Wiley, 1996, pp 309318.
$33 \mathrm{Mu}$ W, Ouyang X, Agarwal A, Zhang L, Long DA, Cruz PE, Roncal CA, Glushakova OY, Chiodo VA, Atkinson MA, Hauswirth WW, Flottel TR, Rodriguez-Iturbe B, Johnson RJ: IL-10 suppresses chemokines, inflammation, and fibrosis in a model of chronic renal disease. J Am Soc Nephrol 2005; 16:36513660 .

34 Floege J, Alpers CE, Burns MW, Pritzl P, Gordon K, Couser WG, Johnson RJ: Glomerular cells, extracellular matrix accumulation, and the development of glomerulosclerosis in the remnant kidney model. Lab Invest 1992;66:485-497.

35 Li P, Longhurst JC: Neural mechanism of electroacupuncture's hypotensive effects. Auton Neurosci 2010;157:24-30.

36 Li P, Tjen-A-Looi S, Longhurst JC: Rostral ventrolateral medullary opioid receptor subtypes in the inhibitory effect of electroacupuncture on reflex autonomic response in cats. Auton Neurosci 2001;89:38-47.

-37 Li M, Tjen-A-Looi SC, Longhurst JC: Electroacupuncture enhances preproenkephalin mRNA expression in rostral ventrolateral medulla of rats. Neurosci Lett 2010;477:6165.

38 Lee HS, Yu YC, Kim ST, Kim KS: Effects of moxibustion on blood pressure and renal function in spontaneously hypertensive rats. Am J Chin Med 1997;25:21-26.

39 Chao DM, Shen LL, Tjen-A-Looi S, Pitsillides KF, Li P, Longhurst JC: Naloxone reverses inhibitory effect of electroacupuncture on sympathetic cardiovascular reflex responses. Am J Physiol 1999;276:21272134.

40 Flachskampf FA, Gallasch J, Gefeller O, Gan J, Mao J, Pfahlberg AB, Wortmann A, Klinghammer L, Pflederer W, Daniel WG: Acupuncture to lower blood pressure. Circulation 2007;115:3121-3129.

41 Ma SX, Ma J, Moise G: Responses of neuronal nitric oxide synthase expression in the brainstem to electroacupuncture Zusanli (ST-36) in rats. Brain Res 2005;1037:70-77.

42 Harris RC, Neilson EG: Toward a unified theory of renal progression. Annu Rev Med 2006;57:365-380.

43 Dugaich AP, Oliveira-Sales EB, Abreu NP, Boim MA, Bergamaschi CT, Campos RR: Role of the rostral ventrolateral medulla in the arterial hypertension in chronic renal failure. Int J Hypertens 2011;2010:219358.

44 Loriga G: Direct renin inhibition: promising treatment in renoprotection? Recent Pat Cardiovasc Drug Discov 2010;5:113-119.

45 Campese VM, Kogosov E, Koss M: Renal afferent denervation prevents the progression of renal disease in the renal ablation model of chronic renal failure in the rat. Am J Kidney Dis 1995;26:861-865

46 Consolim-Colombo FM, Fiorino P: Sympathetic nervous system and hypertension clinical. Rev Bra Hipertens 2005;12:251-255. 
-47 Schlaich MP, Krum H, Sobotka PA: Renal sympathetic nerve ablation: the new frontier in the treatment of hypertension. Curr Hypertens Rep 2010;12:39-46.

-48 Schlaich MP, Socratous F, Hennebry S, Eikelis N, Lambert EA, Straznicky N, Esler MD, Lambert GW: Sympathetic activation in chronic renal failure. J Am Soc Nephrol 2009;20:933-939.

49 Tjen-A-Looi SC, Li P, Longhurst JC: Prolonged inhibition of rostral ventral lateral medullary premotor sympathetic neurons by electroacupuncture in cats. Auton Neurosi 2003;106:119-131.

-50 Moazzami A, Tjen-A-Looi SC, Guo ZL, Longhurst JC: Serotonergic projection from nucleus raphe pallidus to rostral ventrolateral medulla modulates cardiovascular reflex responses during acupuncture. J Appl Physiol 2010;108:1336-1346.

-51 Tjen-A-Looi SC, Li P, Longhurst JC: Midbrain vlPAG inhibits RVLM cardiovascular sympathoexcitatory responses during electroacupuncture. Am J Physiol Heart Circ Physiol 2006;290:H2543-H2553.

-52 Li P, Tjen-A-Looi SC, Guo ZL, Fu LW, Longhurst JC: Long-loop pathways in cardiovascular electroacupuncture responses. J Appl Physiol 2009;106:620-630.
53 Li P, Tjen-A-Looi SC, Longhurst JC: Nucleus raphe pallidus participates in midbrainmedullary cardiovascular sympathoinhibition during electroacupuncture. Am J Physiol Regul Integr Comp Physiol 2010; 299:R1369-R1376.

54 Zhao ZQ: Neural mechanism underlying acupuncture analgesia. Prog Neurobiol 2008;85:355-375.

55 Tjen-A-Looi SC, Li P, Longhurst JC: Role of medullary GABA, opioids, and nociceptin in prolonged inhibition of cardiovascular sympathoexcitatory reflexes during electroacupuncture in cats. Am J Physiol Heart Circ Physiol 2007;293:H3627-H3635.

56 Fu LW, Longhurst JC: Electroacupuncture modulates vlPAG release of GABA through presynaptic cannabinoid CB1 receptors. J Appl Physiol 2009;106:1800-1809.

57 Baylis C, Mitruka B, Deng A: Chronic blockade of nitric oxide synthesis in the rat produces systemic hypertension and glomerular damage. J Clin. Invest 1992;90:278-281.

58 Blum M, Yachnin T, Wollman Y, Chernihovsky T, Peer G, Grosskopf I, Kaplan E, Silverberg D, Cabil S, Iaina A: Low nitric oxide production in patients with chronic renal failure. Nephron 1998;79:265-268.
59 Schmidt RJ, Baylis C: Total nitric oxide production is low in patients with chronic renal disease. Kidney Int 2000;58:1261-1266.

60 Huang YL, Fan MX, Wang J, Li L, Lu N, Cao YX, Shen LL, Zhu DN: Effects of acupuncture on nNOS and iNOS expression in the rostral ventrolateral medulla of stress-induced hypertensive rats. Acupunct Electrother Res 2005;263-273.

61 Kim DD, Pica AM, Durán RG, Durán WN: Acupuncture reduces experimental renovascular hypertension through mechanisms involving nitric oxide synthases. Microcirculation 2006;13:577-585.

62 Hwang HS, Kim YS, Ryu YH, Lee JE, Lee YS, Yang EJ, Lee MS, Choi SM: Electroacupuncture delays hypertension development through enhancing NO/NOS activity in spontaneously hypertensive rats. Evid Based Complement Alternat Med 2008;10:1-6.

63 Huang CL, Tsai PS, Wang TY, Yan LP, Xu HZ, Huang CJ: Acupuncture stimulation of ST-36 (Zusanli) attenuates acute renal but not hepatic injury in lipopolysaccharidestimulated rats. Anesth Analg 2007;646654 